\title{
Does the Intuition of Distinctness Result from Valid Reasoning? - Reply to Kammerer
}

\author{
Karol Polcyn ${ }^{1}$ (1)
}

Accepted: 14 October 2021 / Published online: 6 November 2021

(C) The Author(s) 2021

\begin{abstract}
According to an influential physicalist view, the intuition of distinctness is a cognitive illusion in the sense that it results from fallacious reasoning: we erroneously infer that the referents of phenomenal and physical concepts are different, from the fact that there is a certain difference between our uses of those concepts. (Kammerer, Review of Philosophy and Psychology 10:649-667, 2019) has recently argued, however, that it is psychologically implausible that the intuition of distinctness results from a fallacy: the reasoning process leading to this intuition is, in several psychological respects, similar to valid reasoning and dissimilar to typical fallacies, which gives us a reason to think that this process is a case of valid reasoning. I argue that there are no psychological reasons to think that the process underlying the intuition of distinctness (or at least the crucial part of this process) is a case of valid reasoning. There is, in fact, only one crucial psychological respect in which this process resembles valid reasoning, and although the two processes are similar in one crucial respect, this does not rule out that the intuition of distinctness results from a fallacy, namely, the sort of fallacious reasoning that physicalists have in mind. Furthermore, since the process underlying the intuition of distinctness resembles typical fallacies in one crucial respect, there is a reason to think that the intuition of distinctness does result from a fallacy.
\end{abstract}

\section{Introduction}

The thesis that conscious states are identical with physical states encounters strong intuitive resistance, to which even convinced materialists are not immune. There is something peculiar about the nature of conscious experiences, namely, there is something it is like for subjects to be conscious. For example, there is something it is like for me to see red, and there is something it is like for me to feel pain. Intuitively, however, there is nothing it is like to be a purely physical being. Intuitively, then,

Karol Polcyn

karol.polcyn@gmail.com

1 Institute of Philosophy and Cognitive Science, University of Szczecin, Szczecin, Poland 
physical states do not seem to be the kind of states that have the nature of conscious states.

Many physicalists assume that the intuition of distinctness is a cognitive illusion: we can explain why this intuition arises consistently with assuming that conscious states are, in fact, physical. In particular, many influential physicalists assume that the intuition of distinctness results from fallacious reasoning: when we reflect on the relation between consciousness and physical states and conclude that physicalism is false, we have the tendency to make a kind of reasoning mistake (Balog 2012; Loar 1997; Papineau 1993, 2002, Chapter 6, 2007; Tye 1999). ${ }^{1}$

Kammerer (2019) has recently argued, however, that it is psychologically implausible that the intuition of distinctness results from a fallacy. The crucial psychological features of the process by which we are led to the intuition of distinctness are similar to the crucial psychological features of valid reasoning and dissimilar to the features of typical fallacies. If so, we have a reason to think that the process underlying the intuition of distinctness is a case of valid rather than fallacious reasoning.

Here I argue that Kammerer's argument does not succeed. There are no psychological reasons to think that the process underlying the intuition of distinctness is a case of valid reasoning. So, it is not psychologically implausible that the intuition of distinctness results from a fallacy. In fact, there is a psychological reason to think that the intuition of distinctness does result from a fallacy.

Section 2 outlines the most influential account of the intuition of distinctness as the result of a fallacy. Sections 3 and 4 outline Kammerer's argument for the view that the intuition of distinctness results from valid reasoning. My criticism of Kammerer's account is spelled out in Section 5.

\section{The Intuition of Distinctness as the Result of a Fallacy}

According to the most elaborate and influential account of the intuition of distinctness as the result of a fallacy, developed by Papineau (1993, 2002, Chapter 6, 2007), the fallacy is generated by a certain peculiar feature of phenomenal concepts, the sort of concepts we use when we attend to our own experiences and think about what it is like to undergo them. The relevant feature of those concepts creates the impression that physical concepts, the sort of concepts we use to think about physical states, leave out experiences, from which we infer - fallaciously - that physical concepts simply do not refer to experiences.

What is so peculiar about phenomenal concepts? As Papineau points out, exercising a phenomenal concept typically involves either undergoing the experience being referred to or imagining having that experience. However, imagined recreations of experiences can be regarded as versions of the experience itself, since imagining having an experience is phenomenologically similar to actually having that experience. If so, we can say that our typical exercises of phenomenal concepts always involve a version of the experience being referred to: either the actual experience or

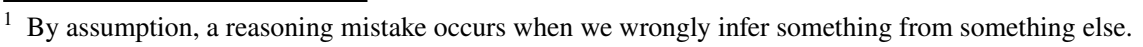


an imaginary recreation of it. In this sense, phenomenal concepts use experiences in order to mention them. ${ }^{2}$

Suppose then that we entertain the hypothesis that a certain type of conscious experience (pain) is identical with a certain type of physical state (c-fibers activation). Our exercise of the phenomenal concept uses or involves a version of the experience being thought about. Because of this, exercising the phenomenal concept feels like having the experience itself. In other words, our exercise of the phenomenal concept activates, as Papineau puts it, the experience being thought about (one or the other version of it). By contrast, the exercise of the physical concept does not activate any version of this experience. If so, there is an intuitive sense in which the physical concept leaves out the experience denoted by the corresponding phenomenal concept: the exercise of the physical concept does not activate any version of that experience. From this observation we then slide to the conclusion that the physical concept simply does not refer to the experience at issue. This is how we become intuitive dualists.

However, if this is how the intuition of distinctness arises, the intuition is the result of a fallacy. There is a sense in which physical concepts leave out experiences: our exercises of physical concepts do not activate the experiences being thought about, by contrast with our exercises of phenomenal concepts. But it does not follow from this that physical concepts do not refer to experiences. Since physical concepts - like most other concepts - do not use their referents, they can well refer to experiences despite not using them. ${ }^{3}$

Call the above physicalist account of the intuition of distinctness the Fallacy Account. The Fallacy Account seems to be an attractive physicalist explanation of why the intuition of dualism is an illusion. According to Kammerer (2019), however, the above account is psychologically implausible. Kammerer argues, on general grounds, that it is psychologically implausible that the intuition of distinctness results from a fallacy: there are psychological reasons to think that the intuition results from valid reasoning. I outline Kammerer's argument in the next section.

\section{The Intuition of Distinctness as the Result of Valid Reasoning}

Kammerer argues that there are three crucial psychological features of the process by which we are led to the intuition of distinctness, and that none of them is similar to the psychological features of typical fallacies; instead, all the three features correspond to the key psychological features of valid reasoning. If so, each of the three features gives us a reason to think that the process by which we are led to

\footnotetext{
${ }^{2}$ By contrast, other concepts do not use or involve the states they mention, as Papineau points out. For example, when I think of being rich or having measles, this doesn't in any sense make me rich or give me measles (Papineau 2002, Chapter 6, 2007).

3 Similar accounts of the intuition of distinctness as the result of a fallacy have been articulated by Balog (2012), Loar (1997) and Tye (1999). Papineau's account is, however, the most elaborate and explicit articulation of the idea that the intuition of distinctness results from a fallacy.
} 
the intuition of distinctness is a case of valid reasoning. Let me briefly outline the details of Kammerer's psychological analysis.

To begin, Kammerer distinguishes two crucial features of conscious experiences: their subjectivity and their qualitativeness. Although Kammerer does not explicitly define those features, I take it that we can characterize them as follows. An experience is subjective in the sense that there is something it is like to have it for the subject of that experience. When I see red or green, for example, there is something it is like to see red or green for me, the subject of experience. A given mental state is a conscious experience precisely in virtue of being subjective. On the other hand, the qualitative character of an experience is what it is like to have that experience for its subject. Qualitative character is what differentiates types of conscious experiences from one another. Thus, what it is like to see red is not the same as what it is like to see green, and that is why the experience of red is not the same as the experience of green. ${ }^{4}$

Kammerer assumes that the subjectivity and qualitativeness of consciousness are responsible for our intuitive resistance to physicalism. When we reflect on our current experiences and come to believe that they are distinct from physical processes, we come to this conclusion precisely by reflecting on the subjectivity and qualitativeness of our experiences. The observation that experiences are subjective and qualitative is what intuitively leads us to the belief that experiences are not identical with physical states. Kammerer identifies three crucial psychological features of this reasoning process and argues that they correspond to the crucial features of valid reasoning.

One psychologically remarkable feature of the process at issue, according to Kammerer, is that it is sustained by careful reflection. Suppose I focus introspectively on my current experience (say, the experience of blue) and consider whether this experience is identical with some electrochemical activity taking place in my visual cortex. The two things strike me as utterly distinct. As Kammerer points out, the more I think introspectively about my current experience, its subjectivity and qualitativeness, the more it seems obvious to me that it cannot be identical with any sort of electrochemical activity. Valid reasoning is also sustained by careful reflection. Consider the following example of valid reasoning leading to a false conclusion. Anne believes, on the basis of what she has been told by other people, that there is nothing in the room but a white chair. When she enters the room, she has a hallucination of a black cat sitting on a white chair. She forms the perceptual judgement that there is a black cat in the room, from which she infers - validly - that it is false that there is nothing in the room but a while chair. As Kammerer points out, Anne's reasoning is sustained by careful reflection. In other words, Anne's conclusion is the product of careful reflection. The more Anne thinks about what she sees in the room, the more it is obvious to her that it cannot be the case that there is nothing in the room but a white chair. By contrast, fallacies are not sustained by careful reflection. Careful reflection does not suppress our tendency to commit

\footnotetext{
${ }^{4}$ For more on the distinction between subjectivity and qualitative character, see Kriegel (2005) and Levine (2001, p. 6-9).
} 
fallacies. Nonetheless, when we make fallacious judgments, our judgments are not the product of careful reflection. ${ }^{5}$ So, to the extent that the process underlying the intuition of distinctness is sustained by careful reflection, it resembles valid reasoning rather than a fallacy. This, according to Kammerer, gives us one reason to think that the process by which we are led to the intuition of distinctness is a case of valid reasoning.

Here is, according to Kammerer, the second crucial psychological feature of the process leading to the intuition of distinctness. When I focus on my current experience and reflect on its subjectivity and qualitativeness, I am not only disposed to believe that it is distinct from physical states - I also find it hard to understand how my experience could be identical with a physical state (e.g., a physical state of my brain). That is, I cannot intelligibly think of the very possibility that my current experience is the same thing as some physical activity in my brain. ${ }^{6}$ Similarly, a certain sense of unintelligibility, according to Kammerer, can be associated with valid reasoning: we do not understand how the conclusions of such reasonings could be false, granted that the premises are true. To go back to Kammerer's earlier example, when Anne concludes, on the basis of her hallucination of a black cat in the room, that it is not true that there is nothing in the room but a white chair, she cannot make sense of the idea that there is nothing in the room but a white chair while there is a black cat. By contrast, no such corresponding sense of unintelligibility can be associated with typical fallacies. For example, the right answer to the question involved in the conjunction fallacy is perfectly intelligible on careful reflection. Thus, the second psychological feature of the process underlying the intuition of distinctness gives us, according to Kammerer, another reason to think that the intuition of distinctness results from valid reasoning.

Finally, here is what Kammerer identifies as the third crucial psychological feature of the process underlying the intuition of distinctness: when I focus on my current experience, different reasoning paths can lead me to the intuitive conclusion that this experience cannot be the same as a physical state. For example, I can focus on the qualitative character of my experience: my experience is qualitative but

\footnotetext{
5 Kammerer considers the "conjunction fallacy" as a classic fallacy studied in psychology of reasoning (Fisk 2004; Kahneman 2012; Tversky and Kahneman 1983). Participants of an experiment are given the following description of a certain person: "Linda is thirty-one years old, single, outspoken, and very bright. She majored in philosophy. As a student, she was deeply concerned with issues of discrimination and social justice, and also participated in antinuclear demonstrations". Subjects are then asked to say which is more probable: (1) Linda is a bank teller or (2) Linda is a bank teller and is active in the feminist movement. As it turns out, the vast majority of people answer that (2) is more likely. But that is a false answer. (2) contains more information - (2) implies (1) but not conversely - and that is why (1) is necessarily more probable than (2). As Kammerer points out, when we reflect carefully, we know that (1) is the right answer. However, even when we reflect carefully, we have the strong tendency to say that (2) is the right answer. This is what makes our mistaken reasoning the case of a fallacy rather than a random error.

6 As Kammerer points out, being strongly disposed to believe that $\mathrm{P}$ and having difficulty understanding how not-P could be true are two different psychological facts. For example, although I am strongly disposed to believe, in the light of my current perceptual evidence, that I have two hands, I have no difficulty understanding how, in spite of what I experience, I may not have two hands (I can picture a possible situation in which I am hallucinating).
} 
physical states are not, therefore my experience cannot be identical with a physical state (by Leibniz's Law). I can then follow the same reasoning by focusing on the subjective character of my experience. I can also reach my conclusion on the basis of epistemological and modal considerations, respectively, namely considerations operative in the knowledge argument (Jackson 1982) and the various modal arguments (Chalmers 1996; Kripke 1980). In this respect, according to Kammerer, the process underlying the intuition of distinctness resembles the case of Anne's valid reasoning, since Anne can follow different paths to conclude, from the fact that there is a black cat in the room, that it cannot be the case that there is nothing in the rom but a white chair. She can take into account the colors of the objects considered (a black object cannot be white) or the kinds of objects (a cat cannot be a chair). By contrast, fallacies, such as the conjunction fallacy, do not involve different reasoning paths; the fallacious conclusion is typically reached in a single and simple step. If this is so, then here again we can see that the process leading to the intuition of distinctness resembles valid reasoning rather than typical fallacies. Kammerer concludes that the third psychological feature of the process underlying the intuition of distinctness gives us one more reason to think that the intuition of distinctness results from valid reasoning.

To sum up, Kammerer argues that the process leading to the intuition of distinctness is, from a psychological point of view, similar to valid reasoning and dissimilar to typical fallacies, and that this gives us a reason to think that this process is a case of valid reasoning. The idea that the intuition of distinctness results from a fallacy is psychologically implausible.

It is reasonable to say that, if the intuition of distinctness results from valid reasoning, the intuition is good evidence that physicalism is false. Kammerer's argument can therefore be seen as a serious challenge for physicalism. ${ }^{7}$

\section{Kammerer's Modified Argument}

I take it that Kammerer's argument requires a certain modification. The need for modification is related to how Kammerer understands the psychological process underlying the intuition of distinctness.

As Kammerer's psychological analysis suggests, there are two steps in the process underlying the intuition of distinctness. I focus introspectively on my current

\footnotetext{
7 Kammerer points out that there are at least two ways in which we can remain physicalists, even if we accept that the intuition of distinctness results from valid reasoning. On the one hand, we may think of consciousness as an illusion: while introspection represents phenomenal properties as having qualitative and subjective nature, the truth may be that there are no such properties (Graziano 2013; Humphrey 2011; Pereboom 2011). On the other hand, we may suppose that, although our introspective judgments are true, we lack the conceptual resources to understand the physical nature of consciousness, and that this is why we are led to believe that conscious states are not identical with physical states (McGinn 1989; Nagel 1974, 2000; Stoljar 2005, 2006). However, both of these views are very controversial, as Kammerer himself points out. On the one hand, it is counterintuitive to abandon realism about phenomenal states. On the other hand, the view that our current physical concepts do not capture all aspects of the 'physical' world is sometimes accused of leading to unstable versions of physicalism, such as neutral monism (Chalmers 2002).
} 
experience and reflect on its subjectivity and qualitativeness. As I reflect on those features, I come to think that they are not physical features so that physical states are neither subjective nor qualitative. That is the first step. Having made this step, I then conclude, from the premise that my experience is subjective and qualitative and the premise that physical states are neither subjective nor qualitative, that my experience is not identical with a physical state. That is the second step.

The second step in the above process is, of course, valid; it is an intuitive application of Leibniz's Law. But what about the first step? Is the kind of process by which I come to judge that subjectivity and qualitativeness are not physical features also a case of valid reasoning? Are there any good reasons to think that it is? I take it that Kammerer's argument should be rearticulated as a response to this question. That is, Kammerer must show that the kind of process by which I come to judge that subjectivity and qualitativeness are not physical features is psychologically similar to the process of valid reasoning and dissimilar to typical fallacies. This would justify the view that this process is also a case of valid reasoning.

Now, Kammerer himself provides such a rearticulation, in addition to his main argument, outlined in the previous section. He argues that the process by which we come to think that subjectivity and qualitativeness are not physical features is similar to a process of valid reasoning in pretty much the same respects as the process by which we come to think that phenomenal states cannot be identical with physical states. First, the process by which we come to think that subjectivity and qualitativeness cannot be physical features is the product of careful reflection. Secondly, we do not understand how subjectivity and qualitativeness, given our introspective grasp of them, could be physical features. Thirdly, different reasoning paths (e.g., reasoning paths appealing to the categorical, modal and epistemological properties of subjectivity and qualitativeness) can lead us to the intuitive conclusion that subjectivity and qualitativeness cannot be physical features. I will focus on this modified version of Kammerer's argument in the discussion below.

\section{Does the Intuition of Distinctness Result from Valid Reasoning?}

Has Kammerer given us good reasons to think that we reason validly when we come to judge that subjectivity and qualitativeness cannot be physical features? In my view, he hasn't. While Kammerer attributes three crucial psychological features to the process at issue and argues that each of them gives us a reason to think that we reason validly when we come to judge that subjectivity and qualitativeness are not physical features, my view is that none of those features gives us such a reason.

Consider the first feature that Kammerer attributes to the process underlying the intuitive judgment that subjectivity and qualitativeness cannot be physical features, namely, the fact that this process is sustained by careful reflection. The more I think introspectively about my current experience, its subjectivity and qualitativeness, the more it seems obvious to me that subjectivity and qualitativeness cannot be entirely physical. No doubt, to the extent that my reasoning is sustained by careful reflection, it resembles valid reasoning, not fallacies. But does this give us a reason to think that my reasoning is valid? Think of the use-mention fallacy, outlined in Section 2. It seems that 
we cannot rule out that I commit this sort of fallacy, even though my reasoning is based on careful reflection. As I focus on my current experience, I entertain the hypothesis that the subjectivity and qualitativeness of my current experience are physical features. In reflecting on the subjectivity and qualitativeness of my current experience, I use the phenomenal concept of my experience. My exercise of this concept activates a version of my experience (namely, the experience itself). By contrast, my exercises of physical concepts do not activate any version of my current experience. There is therefore an intuitive sense in which physical concepts leave out the subjectivity and qualitativeness of my current experience: they leave them out in the sense that my exercises of those concepts do not activate any version of my experience. This observation may then lead me - fallaciously - to the conclusion that physical concepts simply do not refer to the subjective and qualitative aspects of my experience. In effect I may be led to the conclusion that subjectivity and qualitativeness are not physical features. ${ }^{8}$

Is there any good reason to think that careful reflection on my current experience should prevent me from committing the above sort of fallacy? As far as I can see, there is no such reason. By assumption, the use-mention fallacy is generated by the use-mention feature of phenomenal concepts, namely, the fact that our typical uses of phenomenal concepts involve a version of the experience being referred to. But phenomenal concepts are involved in my introspective thinking about the subjectivity and qualitativeness of my current experience. So no matter how much careful my thinking is, the use-mention feature of phenomenal concepts will always create the impression that physical concepts leave out the subjectivity and qualitativeness of my current experience. And it is hard to see how careful reflection on my own experiences could prevent me from sliding, from the above impression, to the corresponding dualist conclusion.

Notice that in order to see that the implication at issue does not hold, I need to reflect carefully on the intuitive sense in which physical concepts leave out the subjectivity and qualitativeness of experiences - more specifically, I need to reflect on how the intuitive sense that physical concepts leave out those features can be consistent with assuming that physical concepts refer to them. When I engage in this sort of reflection, I can realize that I will have the intuitive sense that physical concepts leave out the subjectivity and qualitativeness of experiences, even if physical concepts refer to those features. After all, the relevant phenomenological difference between our uses of phenomenal and physical concepts will create the intuitive sense that physical concepts leave out subjectivity and qualitativeness, even if physical concepts do refer to subjectivity and qualitativeness. But this sort of reflection is, obviously, not involved in my reflection on my current experiences as such. I can reflect very carefully on my current experiences, their subjectivity and qualitativeness without reflecting on how the intuitive sense that physical concepts leave out those features can be consistent with physicalism. Careful reflection on the subjectivity and qualitativeness of my own experiences, on the one hand, and careful reflection on the intuitive sense in which physical concepts leave out those

\footnotetext{
${ }^{8}$ Let me emphasize that I am not saying here that careful reflection on the hypothetical identity of phenomenal and physical states sustains the use-mention fallacy. I do not think that careful reflection sustains the use-mention fallacy. Rather, my point is that we cannot expect that careful reflection on the hypothetical identity of phenomenal and physical states will prevent us from committing the fallacy at issue.
} 
features, on the other, are two different psychological processes. For this reason, one cannot expect careful reflection on my own experiences to enable me to see that the implication from the intuitive sense that physical concepts leave out subjectivity and qualitativeness to the conclusion that physical concepts do not refer to those features does not hold. ${ }^{9}$

Interestingly, Kammerer suggests that there is a good reason to expect that careful reflection on our current experiences (and the corresponding physical states) should prevent us from committing the use-mention fallacy after all. As Kammerer assumes, the fallacy consists in projecting the relevant phenomenological difference between our uses of phenomenal and physical concepts (namely, the fact that our exercises of physical concepts, by contrast with our exercises of phenomenal concepts, do not activate any version of the experience being thought about) on the referents of those concepts; correspondingly, in our present context, the fallacy consists in inferring, from the relevant phenomenological difference between our exercises of phenomenal and physical concepts, that physical concepts do not refer to the subjective and qualitative aspects of experiences. But there is a good reason to expect, according to Kammerer, that careful reflection should enable us to avoid making this sort of projection. Careful reflection leads to an increase in introspective attention - it leads us to paying closer attention to our thinking with phenomenal concepts and our thinking with physical concepts - and for this reason, it makes it more salient and obvious to us that our exercises of physical concepts, by contrast with our exercises of phenomenal concepts, do not activate any version of the experience being thought about. But when this crucial phenomenological difference between our uses of phenomenal and physical concepts becomes more salient and obvious to us, we should be able to "tear apart what belongs to the use of the concepts and what belongs to their referents" (Kammerer 2019, p. 664). That is, we should be able to see that the relevant difference between our exercises of phenomenal and physical concepts does not entail that the referents of those concepts are different - that physical concepts do not refer to experiences. Applying Kammerer's point to our context, we should be able to see that it does not follow, from the relevant difference between our exercises of phenomenal and physical concepts, that physical concepts do not refer to the subjective and qualitative aspects of experiences. ${ }^{1011}$

\footnotetext{
9 Similarly, there is no reason to think that careful reflection on my current experience should prevent me from committing the use-mention fallacy understood as the sort of reasoning by which we come directly to the conclusion that physical concepts do not refer to experiences.

${ }^{10}$ Kammerer makes the point outlined here in response to the objection that careful reflection actually sustains the use-mention fallacy by making it more salient to us - through increased introspective attention - that our exercises of physical concepts, by contrast with our exercises of the corresponding phenomenal concepts, do not activate any version of the experience being thought about. As Kammerer argues, careful reflection does not sustain the use-mention fallacy: although careful reflection intensifies the relevant phenomenological difference between our uses of phenomenal and physical concepts, it does not sustain the fallacious projection of this difference on the referents of phenomenal and physical concepts. Kammerer then goes on to say that we should expect careful reflection to actually prevent us from committing the use-mention fallacy, for the reasons outlined here.

11 Notice that here Kammerer articulates a direct argument against the Fallacy Account as one specific account of the intuition of distinctness. His initial intention was to give a general argument against the view that the intuition of distinctness results from a fallacy. Here he departs from this general strategy to argue directly against one specific account of why the intuition of distinctness results from a fallacy.
} 
Notice that the more salient and obvious it becomes to us that there is the relevant phenomenological difference between our uses of phenomenal and physical concepts, the more salient it becomes to us that there is the intuitive sense in which physical concepts leave out the subjectivity and qualitativeness of experiences, namely, that physical concepts leave out the subjectivity and qualitativeness of experiences in the sense that our exercises of those concepts, by contrast with our exercises of phenomenal concepts, do not activate any version of the experience being thought about. Kammerer's view thus comes down to the point that when it becomes more obvious and salient to us that there is the intuitive sense in which physical concepts leave out the subjectivity and qualitativeness of experiences, we should be able to see that it does not follow, from the fact that there is this intuitive sense, that physical concepts do not refer to the subjectivity and qualitativeness of experiences.

Kammerer gives no reason for the above view; he finds it obvious. But it should be clear, in the light of what we argued above, that Kammerer's view cannot be right. To see that there is no implication, from the intuitive sense that physical concepts leave out the subjective and qualitative aspects of experiences, to the conclusion that physical concepts do not refer to those aspects, I need to engage in careful reflection on the intuitive sense in which physical concepts leave out the subjectivity and qualitativeness of experiences. More specifically, I need to reflect on how the intuitive sense that physical concepts leave out those features can be consistent with assuming that physical concepts refer to them. But this sort of reflection is, obviously, not involved in my having the strong intuitive sense that physical concepts leave out the subjectivity and qualitativeness of my own experiences. I can have the strong intuitive sense that physical concepts leave out the subjectivity and qualitativeness of my experiences, even when I do not reflect on this intuitive sense and do not consider whether or not it follows, from the fact that there is this sense, that physical concepts do not refer to subjectivity and qualitativeness. Having the strong intuitive sense that physical concepts leave out the subjectivity and qualitativeness of experiences, on the one hand, and careful reflection on this intuitive sense, on the other, are two different psychological processes. If this is so, then I cannot see that it does not follow that physical concepts do not refer to the subjectivity and qualitativeness of experiences, from the fact that there is the intuitive sense in which they leave them out, simply in virtue of noticing more clearly that physical concepts do leave out subjectivity and qualitativeness in the intuitive sense at issue.

To sum up, we cannot agree with Kammerer's way of defending the claim that we should be able to see upon careful reflection on our current experiences that it does not follow, from the relevant phenomenological difference between our uses of phenomenal and physical concepts, that physical concepts do not refer to the subjective and qualitative aspects of experiences. It is true that my careful reflection on my own experiences will intensify the relevant difference between our uses of phenomenal and physical concepts, as Kammerer himself admits. But this will not, contrary to what Kammerer claims, enable me to see that the difference at issue does not imply that physical concepts do not refer to the subjective and qualitative aspects of experiences. I will be able to see more clearly that physical concepts leave out the subjectivity and qualitativeness of experiences, but this will not 
enable me to see - for the reason articulated above - that the implication at issue does not hold. ${ }^{12}$

If Kammerer's strategy does not work, it is hard to see how else one could justify the claim that, when we reflect carefully on our current experiences, we should be able to see that the intuitive sense that physical concepts leave out subjectivity and qualitativeness does not lead to the conclusion that subjectivity and qualitativeness are not physical features. So, I conclude that there is no reason to expect that careful reflection on my own experiences should prevent me from committing the use-mention fallacy. This means that the first step of Kammerer's main argument fails: the fact that the process leading me to the intuition of distinctness is sustained by my careful reflection on my own experiences gives us no reason to think that this process is a case of valid reasoning. ${ }^{13}$

Consider the second psychological feature that Kammerer attributes to the process by which we are led to judge that subjectivity and qualitativeness are not physical features. When I focus on my current experience and reflect on its qualitativeness and subjectivity, I am not only disposed to believe that subjectivity and qualitativeness are not, as a matter of facts, physical features - I also find it hard to understand how subjectivity and qualitativeness could be physical features. That is, I cannot intelligibly think of the very possibility that subjectivity and qualitativeness are physical features, e.g., physical features of my brain. In other words, I cannot picture a possible situation in which my conscious experience is entirely physical while being qualitative and subjective. Does this give us a reason to think that we reason validly when we are led to think that subjectivity and qualitativeness cannot be physical features? Kammerer argues that it does, because he thinks that our difficulty in understanding how subjectivity and qualitativeness could be physical features corresponds to an important feature of valid reasoning, namely, to the fact that when we reason validly, we do not understand how the conclusions of our reasonings could be false, granted that the premises are true. In reply, I can see two problems with this argument.

\footnotetext{
12 A proponent of the Fallacy Account might argue further that the idea that careful reflection makes it more salient that physical concepts leave out the subjectivity and qualitativeness of experiences can well be accommodated by the Fallacy Account: when it becomes more salient and obvious to us that physical concepts leave out those features, it becomes even more salient and obvious to us at an intuitive level that those features are not physical features.

13 As I mentioned in note 10, Kammerer discusses a related but stronger objection to his account of the intuition of distinctness, namely, that careful reflection sustains the use-mention fallacy, and argues in response that this objection fails. I agree with Kammerer that careful reflection does not sustain the use-mention fallacy (even though I do not agree with Kammerer's further point that careful reflection may prevent us from committing the use-mention fallacy). Still, I have shown here that the idea that the intuition of distinctness results from the use-mention fallacy raises a problem for Kammerer's account, even if we agree that careful reflection does not sustain the use-mention fallacy: the mere fact that careful reflection cannot prevent us from committing the use-mention fallacy undermines Kammerer's claim that, insofar as the process underlying the intuition of distinctness is sustained by careful reflection, we have a reason to think that this process is a case of valid reasoning.
} 
First, while Kammerer assumes that our difficulty in understanding how subjectivity and qualitativeness could be physical features is a psychological feature of the process by which we are led to judge that subjectivity and qualitativeness cannot be physical features, this assumption does not seem to be right. As we saw, Kammerer himself argues that being strongly disposed to believe that $\mathrm{P}$ and having difficulty understanding how not-P could be true are two different psychological facts in the sense that the former does not imply the latter. According to Kammerer, there is no such implication, because we can think of cases where one is strongly disposed to believe that $\mathrm{P}$, even though one has no difficulty understanding how not-P could be the case (see note 6). Applying this point to our case, the fact that I do not understand how subjectivity and qualitativeness could be physical features is not implied by the fact that I am strongly disposed to believe that subjectivity and qualitativeness are not physical features. But this means that our difficulty in understanding how subjectivity and qualitativeness could be physical features is not a psychological feature of the process leading us to judge that subjectivity and qualitativeness are not physical features.

One might argue that there is a sense in which the two facts considered here - our difficulty in understanding how subjectivity and qualitativeness could be physical features and our disposition to believe that subjectivity and qualitativeness are not, as a matter of facts, physical features - are nonetheless psychologically related to each other: both are facts about our cognitive assessment of the idea that subjectivity and qualitativeness are physical features, based on our introspective grasp of our own experiences. However, even if there is this sort of relation, there is another problem with Kammerer's argument. By assumption, the intuitive sense that I do not understand how subjectivity and qualitativeness could be physical features is not implied by my intuitive belief that subjectivity and qualitativeness are not physical features. In other words, although I do not accept at an intuitive level that subjectivity and qualitativeness are physical features, this does not explain why I do not understand how subjectivity and qualitativeness could be physical features. On the other hand, notice that when we reason validly, we do not understand how the conclusions of our reasonings could be false, granted that the premises are true, because we do not accept that the conclusions of our reasonings could be false. When we reason validly, we assume that our conclusions cannot be false if the premises are true. Thus, we do not accept that the conclusions of our valid reasonings could be false, granted that the premises are true, and this is why we do not understand how the conclusions of our valid reasonings could be false. Think of Kammerer's example of Anne who concludes, on the basis of her hallucination of a black cat, that it cannot be the case that there is nothing in the room but a white chair. Anne does not accept that the conclusion of her reasoning could be false - there is a contradiction between the claim that there is nothing in the room but a white chair and Anne's belief that there is a black cat in the room - and this explains why she cannot understand how her conclusion could be false. In short, there is a certain obvious way of explaining why Anne does not understand how the conclusion of her reasoning could be false. But it is not possible to give a similar explanation of why we intuitively find it so difficult to understand how subjectivity and qualitativeness could be physical features. If this is so, then the fact that we do not understand how subjectivity and qualitativeness could be 
physical features gives us no reason to think that we reason validly when we come to judge that subjectivity and qualitativeness are not physical features. ${ }^{14}$

Consider now the third psychological feature that Kammerer attributes to the process underlying the judgment that subjectivity and qualitativeness are not physical features: when I focus on my current experience and reflect on its subjectivity and qualitativeness, different reasoning paths - involving considerations on the categorical, modal or epistemological properties of subjectivity and qualitativeness - can lead me to the intuitive conclusion that subjectivity and qualitativeness are not physical features. Does this give us a reason to think that the process at issue is a case of valid reasoning? Kammerer argues that it does on the grounds that some cases of valid reasoning, such as Anne's reasoning, can also be based on different reasoning paths. In reply, it is arguable that Kammerer's conclusion is again unjustified.

The problem is that there is really no similarity between the structure of Anne's reasoning and the fact that different reasoning paths, based on different theoretical considerations, can lead me to the conclusion that subjectivity and qualitativeness are not physical features. In the case of Anne's reasoning, one can identify different reasoning paths leading to the same conclusion (it is not the case that there is nothing in the room but a white chair) from one and the same premise (there is a black cat in the room). The relevant reasoning paths go as follows: "There is a black cat in the room - but nothing can be both a cat and a chair - therefore it is not the case that there is nothing in the room but a white chair", and "There is a black cat in the room - but nothing can be both black and white - therefore it is not the case that there is nothing in the room but a white chair". On the other hand, different reasoning paths leading me to the conclusion that subjectivity and qualitativeness are not physical features will originate from different assumptions. For example, one reasoning path will originate from the relevant epistemological assumption (e.g., the assumption that I can know all the physical facts without knowing what it is like to have such

\footnotetext{
14 As a reviewer points out, there are cases when people deny the conclusions of valid arguments despite accepting the premises. For example, consider the argument that since God is omnipotent and there is evil in the world, God is not benevolent. The argument is valid. But, as the reviewer points out, some religious thinkers would accept the premises of the above argument and yet deny the conclusion while recognizing that they do not fully understand how it is possible for the premises to be true and the conclusion to be false. To such thinkers God is both omnipotent and benevolent despite that fact there is evil in the world, although it remains a mystery how this situation could obtain. Here then we have a case where one does not understand how the conclusion of a valid argument could be false, granted that the premises are true, while accepting that the conclusion is, indeed, false. One might then argue on this basis that my response to Kammerer does not work: the fact that we do not understand how the conclusion of a valid argument could be false does not psychologically stem (at least, not always) from us rejecting the claim that the conclusion could be false. In reply, it is not difficult to see, however, that the case of religious thinkers is not really a counterexample to the point I have made in response to Kammerer. My point is restricted to cases where one goes through the process of valid reasoning: when we reason validly, we do not understand how the conclusions of our reasonings could be false, granted that the premises are true, precisely because we do not accept that the conclusions could be false. The case of religious thinkers, on the other hand, is not a case where one reasons validly. If I reason validly, I will infer that God is not benevolent, from the premises that God is omnipotent and that there is evil in the world. Thus, if I reason validly, I will not deny the above conclusion (that God is not benevolent) if I accept the premises. If, on the other hand, one denies the conclusion at issue despite accepting the premises, which is the case of our religious thinkers, one does not reason validly.
} 
an such an experience), and another reasoning path will originate from the relevant modal assumption (e.g., the assumption that I cannot rule out a priori that the world is a zombie world). In other words, when I appeal to epistemological considerations, I will infer my dualist conclusion from the relevant epistemological assumption, and when I appeal to modal considerations, I will infer my dualist conclusion from the relevant modal assumption. Of course, Anne's reasoning paths can also be said to involve different (implicit) assumptions. For example, one of the reasoning paths available to her relies on an implicit assumption regarding incompatible type-properties of objects (a cat cannot be a chair), whereas another reasoning path relies on an implicit assumption regarding incompatible color-properties of objects (black objects cannot be white). ${ }^{15}$ But the crucial point is that those assumptions function as different inference rules - Anne uses them to infer her conclusion from the assumption that there is a black cat in the room. The initial assumption - one from which Anne's reasoning originates - is always the same. By contrast, reasoning paths leading to the dualist conclusion involve different initial assumptions, not just different inference rules. Anne's reasoning paths are simply different ways of getting to a certain conclusion from one and the same premise. By contrast, reasoning paths leading to the dualist conclusion - to the extent that they originate from different assumptions - are separate arguments. So, the plurality of reasoning paths leading to the intuition of distinctness does not really correspond to the plurality of reasoning paths available to Anne. Even though I can reach the intuition of distinctness on the basis of different theoretical considerations, this gives me no good reason to think that my reasoning is valid.

Now, we have assumed, following Kammerer, that the reasoning process leading to the intuition of distinctness is a sort of process that is based on theoretical considerations. However, it is arguable that there is also a separate psychological process leading to the intuition of distinctness independently of theoretical considerations. When I reach the intuition of distinctness in this second way, I come to judge that subjectivity and qualitativeness are not physical features solely on the basis of my introspective grasp of my current experience, without appealing to any theoretical considerations on subjectivity and qualitativeness, such as modal or epistemological considerations. ${ }^{16}$ The remaining question is whether this process has a psychological feature corresponding to the fact that Anne's reasoning can be based on different reasoning paths. If the process at issue has such a psychological feature, this would give us a reason to think that this process is a case of valid reasoning.

In reply, I do not think that different reasoning paths can lead me to the judgment that subjectivity and qualitativeness are not physical features if I reason simply from my introspective grasp of the subjectivity and qualitativeness of my current experience. When I simply focus on my current experience and reflect on its subjectivity and qualitativeness, there is a sense in which different reasoning paths can lead me

\footnotetext{
15 Thanks to an anonymous reviewer for clarifying this.

${ }^{16}$ We might think of the premise of this intuitive reasoning as follows: "Subjectivity and qualitativeness are the features of my current experience, revealed to me through introspection". When I focus on my current experience and come to judge, independently of theoretical considerations, that subjectivity and qualitativeness are not physical features, I come to this conclusion solely on the basis of the above premise.
} 
to the conclusion that my current experience is not a physical state, as Kammerer himself points out. On the one hand, by focusing on the qualitative character of my current experience, I may come to think that qualitativeness is not a physical feature, and then I can infer further, from the premise that my experience is qualitative and the premise that qualitativeness is not a physical feature, that my experience is not a physical state. On the other hand, I can follow the same reasoning by focusing on the subjective character of my current experience. However, one can hardly make sense of the idea that different reasoning paths can lead me, in the case considered here, to the judgment that qualitativeness and subjectivity as such are not physical features. When I come to think that qualitativeness is not a physical feature, simply by focusing on the qualitative character of my current experience, or when I come to think that subjectivity is not a physical feature, simply by focusing on the subjective character of my current experience, I do not seem to be in a situation in which I can follow different reasoning paths. In the end, we have at this point no good reason to think that the reasoning process leading to the intuition of distinctness independently of theoretical considerations is a case of valid reasoning, either.

To sum up, we have considered three psychological features that Kammerer attributes to the process underlying the intuitive judgment that subjectivity and qualitativeness are not physical features. As it turns out, none of them gives us a reason to think that this process is a case of valid reasoning. First, although the process at issue resembles the process of valid reasoning in being sustained by careful reflection, there is no good reason to expect that careful reflection will prevent us from committing the use-mention fallacy when we come to judge that subjectivity and qualitativeness are not physical features. Second, while we can hardly understand how the content of our intuitive judgment could be false - how subjectivity and qualitativeness could be physical features - we cannot explain why we do not understand this in a similar way in which we can explain why we do not understand how the conclusions of our valid reasonings could be false. Third, while the conclusion that subjectivity and qualitativeness are not physical features can be reached through several different arguments, based on different theoretical considerations, the availability of such different arguments does not correspond to the way in which some cases of valid reasoning, such as Anne's reasoning, can be based on different reasoning paths. Further, no different reasoning paths are involved in the process that leads us to the intuition of distinctness independently of theoretical considerations. If all of this is correct, there is no reason to think that the process underlying the intuition of distinctness (whether based on theoretical considerations or not) is a case of valid reasoning.

Here is another way to summarize our results. The process leading us to judge that subjectivity and qualitativeness are not physical features is, from a psychological point of view, quite different from the process of valid reasoning. On the one hand, although we intuitively do not understand how subjectivity and qualitativeness could be physical features, we cannot explain why we do not understand this in a similar way in which we can explain why we do not understand how the conclusions of our valid reasonings could be false. On the other hand, while different reasoning paths can link premises and conclusions in standard cases of valid reasoning, such as Anne's reasoning, no such corresponding feature can be attributed to the process 
by which we come to think that subjectivity and qualitativeness are not physical features. The process at issue resembles valid reasoning only to the extent that it sustained by careful reflection. But this psychological feature gives us no reason to think that we reason validly when we come to think that subjectivity and qualitativeness are not physical features. So, again, the conclusion to draw is that there is simply no good reason to think that the process at issue is a case of valid reasoning.

Given that there is no psychological reason to think that we reason validly when we come to think that subjectivity and qualitativeness are not physical features, it is not psychologically implausible that our reasoning is fallacious. In effect it is not psychologically implausible to suppose that the intuition of distinctness results from a fallacy.

In fact, there is one further conclusion to be drawn from our considerations. As we argued, we cannot really identify different reasoning paths in the psychological process that leads us to dualism independently of theoretical considerations. That is, there aren't any different reasoning paths that can lead us to judge that subjectivity and qualitativeness are not physical features if our reasoning is based solely on our introspective reflection on our current experience. This means that when we come to judge that subjectivity and qualitativeness are not physical features, simply by way of focusing on our current experience and reflecting on its subjectivity and qualitativeness, we do it in a single and simple step, or perhaps by way of combining two single steps, one leading to the judgment that subjectivity is not a physical feature and another leading to the judgment that qualitativeness is not a physical feature. In this respect, then, the psychological process at issue is similar to typical fallacies, such as the conjunction fallacy. This gives us a reason to think that this process is a fallacy rather than a case of valid reasoning. In effect there is a good psychological reason to think that the intuition of distinctness results from a fallacy.

Acknowledgements I would like to thank two anonymous reviewers for helpful comments and suggestions.

Funding Financial support was provided by National Science Centre, Poland (UMO-2017/25/B/ HS1/02986).

\section{Declarations}

Conflicts of Interest The Author has no conflicts of interest to declare that are relevant to the content of this article.

Open Access This article is licensed under a Creative Commons Attribution 4.0 International License, which permits use, sharing, adaptation, distribution and reproduction in any medium or format, as long as you give appropriate credit to the original author(s) and the source, provide a link to the Creative Commons licence, and indicate if changes were made. The images or other third party material in this article are included in the article's Creative Commons licence, unless indicated otherwise in a credit line to the material. If material is not included in the article's Creative Commons licence and your intended use is not permitted by statutory regulation or exceeds the permitted use, you will need to obtain permission directly from the copyright holder. To view a copy of this licence, visit http://creativecommons.org/licen ses/by/4.0/. 


\section{References}

Balog, Katalin. 2012. Acquaintance and the mind-body problem. In New perspectives on type identity: The mental and the physical, ed. Christopher Hill and Simone Gozzano, 16-43. Cambridge: Cambridge University Press.

Chalmers, David. 1996. The conscious mind: In search of a fundamental theory. New York: Oxford University Press.

Chalmers, David. 2002. Consciousness and its place in nature. In Philosophy of mind: classical and contemporary readings, ed. David Chalmers, 247-272. New York: Oxford University Press.

Fisk, John. 2004. Conjunction fallacy. In Cognitive illusions. A handbook on fallacies and biases in thinking, judgment and memory, ed. Rudiger Pohl, 23-42. Hove, East Sussex: Psychology Press.

Graziano, Michael. 2013. Consciousness and the social brain. Oxford: Oxford University Press.

Humphrey, Nicholas. 2011. Soul Dust: The Magic of Consciousness. Princeton: Princeton University Press.

Jackson, Frank. 1982. Epiphenomenal qualia. The Philosophical Quarterly 32: 127-136.

Kahneman, Daniel. 2012. Thinking, fast and slow. Penguin.

Kammerer, Francois. 2019. Does the explanatory gap rest on a fallacy? Review of Philosophy and Psychology 10: 649-667.

Kriegel, Uriah. 2005. Naturalizing subjective character. Philosophy and Phenomenological Research 71: 23-56.

Kripke, Saul. 1980. Naming and necessity. Cambridge, MA: Harvard University Press.

Levine, Joseph. 2001. Purple haze: The puzzle of consciousness. Oxford: Oxford University Press.

Loar, Brian. 1997. Phenomenal states. In The nature of consciousness, ed. Ned Block, Owen Flanagan, and Güven. Güzeldere, 597-616. Cambridge, MA: MIT Press.

McGinn, Colin. 1989. Can we solve the mind-body problem? Mind 98: 349-366.

Nagel, Thomas. 1974. What is it like to be a bat? Philosophical Review 83: 435-450.

Nagel, Thomas. 2000. The psychophysical nexus. In New essays on the a priori, ed. Paul Boghossian and Christopher Peacocke, 432-471. Oxford: Clarendon Press.

Papineau, David. 1993. Physicalism, consciousness, and the antipathetic fallacy. Australasian Journal of Philosophy 71: 169-183.

Papineau, David. 2002. Thinking about consciousness. Oxford: Oxford University Press.

Papineau, David. 2007. Phenomenal and perceptual concepts. In Phenomenal concepts and phenomenal knowledge: New essays on consciousness and physicalism, ed. Torin Alter and Sven Walter, 111144. Oxford: Oxford University Press.

Pereboom, Derk. 2011. Consciousness and the prospects of physicalism. Oxford University Press.

Stoljar, Daniel. 2005. Physicalism and phenomenal concepts. Mind and Language 20: 296-302.

Stoljar, Daniel. 2006. Ignorance and imagination: The epistemic origin of the problem of consciousness. Oxford: Oxford University Press.

Tversky, Amos, and Daniel. Kahneman. 1983. Extensional versus intuitive reasoning: The conjunction fallacy in probability judgment. Psychological Review 90: 293-315.

Tye, Michael. 1999. Phenomenal consciousness: The explanatory gap as a cognitive illusion. Mind 108: $702-725$.

Publisher's Note Springer Nature remains neutral with regard to jurisdictional claims in published maps and institutional affiliations. 